\title{
A Transient Cosimulation Approach to Performance Analysis of Hybrid Excited Doubly Salient Machine Considering Indirect Field-Circuit Coupling
}

\author{
Xiaoyong Zhu ${ }^{1}$, Ming Cheng ${ }^{1}$, Wenxiang Zhao ${ }^{1}$, Chunhua $\mathrm{Liu}^{2}$, and K. T. Chau ${ }^{2}$ \\ ${ }^{1}$ School of Electrical Engineering, Southeast University, Nanjing 210096, China \\ ${ }^{2}$ Department of Electrical and Electronic Engineering, The University of Hong Kong, Hong Kong, China
}

\begin{abstract}
This paper presents a hybrid excited doubly salient (HEDS) machine, which can be used as the integrated starter-generator (ISG) for modern automobile and hybrid electric vehicles (HEVs). The key of the proposed machine is to incorporate both direct current (de) field windings and permanent magnets (PMs) in the stator, hence, offering a compact arrangement of hybrid field excitations, while the rotor is simply composed of salient poles without windings or PMs. The air-gap flux can be strengthened or weakened with a reasonable dc field current so that the electromagnetic torque and induced electromotive force (EMF) can be effectively regulated. To predict the electromagnetic performances of the machine more accurately, a new transient cosimulation approach considering indirect field-circuit coupling is proposed. Experimental results on a prototype machine have been given to verify the technique. The method is not only more efficient and flexible, but also more accurate and stable, which can also be used in analyzing other electric machine and drive systems.
\end{abstract}

Index Terms-Cosimulation, doubly salient machine, finite element analysis (FEA), hybrid excited machine, integrated startergenerator (ISG).

\section{INTRODUCTION}

$\mathbf{I}$ NSTEAD of using separate machines for cold cranking and battery charging, the concept of integrated starter-generator (ISG) is becoming attractive for modern automobiles and hybrid electric vehicles (HEVs). Because of the requirements of very high starting torque (up to four times the rated torque) for cold cranking, as well as constant output voltage over a very wide speed range ( $25 \%$ to four times the base speed) for battery charging, the design of this ISG is challenging [1]. Recently, a permanent magnet (PM) synchronous machine has been utilized for ISG application, since it inherently offers high torque density and high efficiency. However, because of uncontrollable PM flux, it cannot maintain high efficiency or constant output voltage over the wide speed range. To enable flux control, the idea of stator-doubly-fed doubly-salient (SDFDS) machine has been proposed [2], in which a dc field winding replaces the PMs to facilitate flux control and online efficiency optimization. However, this topology inevitably needs high-field winding magnetomotive force (MMF) to realize the desired flux linkage, hence degrading the machine efficiency.

In this paper, a hybrid excited doubly salient (HEDS) machine is proposed for automotive engines. The machine topology and flux control principle are introduced in Section II. In order to predict the electromagnetic performances of the HEDS machine more accurately, a new transient cosimulation approach is proposed, in which a transient 2-D finite element analysis (FEA) is coupled with circuit simulation. The principle and coupling mechanism are introduced in details in Section III. By using the new cosimulation technique, the corresponding electromagnetic performances are predicted in Section IV. Moreover, a 12/8pole HEDS machine has been designed and built for evaluation. Experimental results of the prototype machine are given to

Digital Object Identifier 10.1109/TMAG.2007.893318

Color versions of one or more of the figures in this paper are available online at http://ieeexplore.ieee.org.

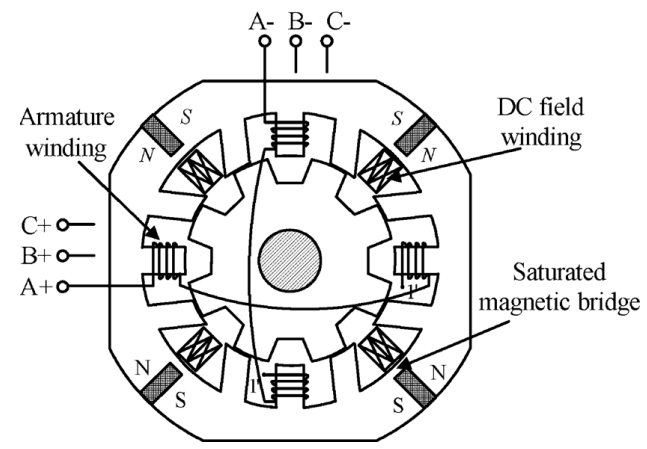

Fig. 1. Cross-section of the proposed HEDS machine.

verify the proposed new cosimulation approach. Finally, some conclusions are drawn.

\section{MACHINE DESCRIPTION AND FLUX CONTROL PRINCIPLE}

Fig. 1 shows the structure of a three-phase 12/8-pole HEDS machine. There are four pieces of PMs in the stator. The machine consists of two types of stator windings: a three-phase concentrated armature winding and a dc field winding. The function of the armature winding is the same as that for an SDFDS machine, whereas the dc field winding works as a tool for flux control and efficiency optimization.

\section{Coupling Principle of the Cosimulation Method}

In this paper, the proposed new cosimulation of indirect coupling method provides the possibility of system level simulation, where integrating the transient magnetic solver of Maxwell2D ${ }^{\circledR}$ into the system circuit simulator, Simplorer ${ }^{\circledR}$.

Fig. 2 illustrates the general flow-chart of the cosimulation method. On the FEA side, at each time step, the solutions of the HEDS machine model are extracted from Maxwell2D at first, and then the coupling inductance matrix and induced voltages together with the winding currents are sent to the circuit 


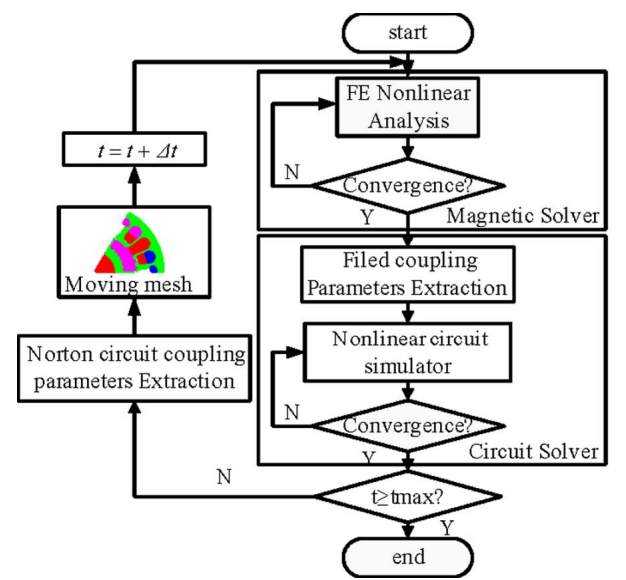

Fig. 2. General flow-chart of the cosimulation method.

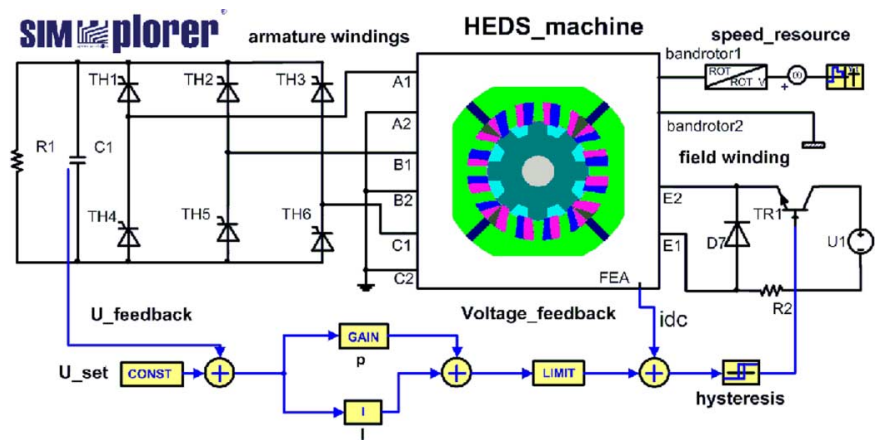

Fig. 3. Integrated finite element/circuit model.

simulator of Simplorer. After FEA at each time step, the FEA system's coefficient matrix is frozen. On the circuit simulation side, these coupling coefficients are used to perform circuit simulation in terms of a smaller circuit time step, hence extracting the Norton equivalent conductance matrix and the source currents flowing into the windings through coupling nodes from the circuit simulator of Simplorer. Maxwell2D converts these Norton circuit coupling parameters to a loop matrix and solves the finite element equations for the next Simplorer time step. A reasonable time step $\Delta T$ is very important to keep the accuracy of the cosimulation method. When the $\Delta T$ is set to $1 \mu \mathrm{s}$ in the HEDS machine system, the cosimulation can be to preserve the required convergence.

The coupled-field analysis [3] is becoming attractive since it can study the interaction between different fields, including electromagnetics, thermal, and mechanics. As this coupled-field analysis is essentially based on FEA, it is possible to incorporate it into the proposed cosimulation approach so that the performance analysis will become more comprehensive.

\section{Performance ANALysis AND Verification}

The newly designed and built HEDS machine is analyzed by using the transient cosimulation method. For comparing the accuracy of the new method and traditional methods, results of both the equivalent magnetic circuit model and a common static FEA are also given.

Fig. 3 shows the proposed 12/8-pole HEDS machine, which is cosimulated by Maxwell2D with Simplorer. From the figure, the HEDS machine model created by Maxwell2D is effectively



Fig. 4. Predicted back-EMF waveforms and the measured one.

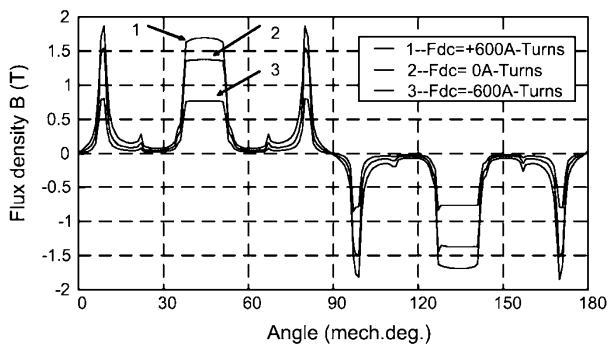

Fig. 5. Radial air-gap flux density distributions with different field excitations.

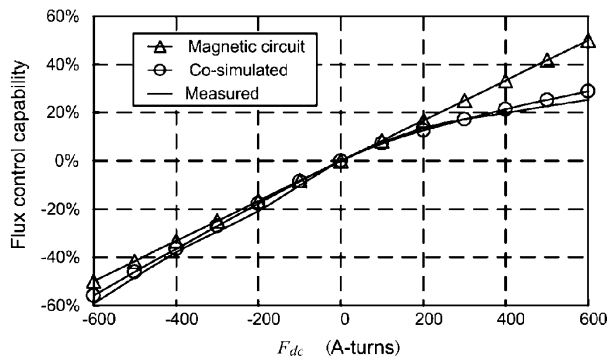

Fig. 6. Flux control capability at different dc field ampere turns.

linked and integrated in the Simplorer project. It should be noted that the electrical pins of the machine, including three-phase armature windings and dc field winding, are available, which are very conveniently connected with external circuit components. On the mechanical side, a velocity source or load can be connected to the machine directly, where inertia and/or damping can be considered in a simple way.

By using the cosimulation method, when a velocity source is given to the machine, it works as a generator, and then the transient back-electromotive force (back-EMF) of no-load at different speeds can be obtained through a voltage meter easily. Fig. 4 shows the cosimulated and measured back-EMFs at the speed of $1000 \mathrm{r} / \mathrm{min}$. For comparison, the result of common FEA is also shown in the figure. In general, the two computational methods agree with the measurement. Because the end resistance and end leakage inductances have been considered in the external circuit of the cosimulation model, the cosimulation method takes advantages of higher accuracy.

To assess the level of the flux control capability, a flux control coefficient $\alpha$ can be defined as follows:

$$
\alpha=\left(B_{\delta}-B_{\delta 0}\right) / B_{\delta 0} \times 100 \%
$$

where $B_{\delta 0}$ is the flux density of the air-gap without dc field current, and $B_{\delta}$ is the flux density at different dc field currents. Fig. 5 shows the flux density distributions under different dc 

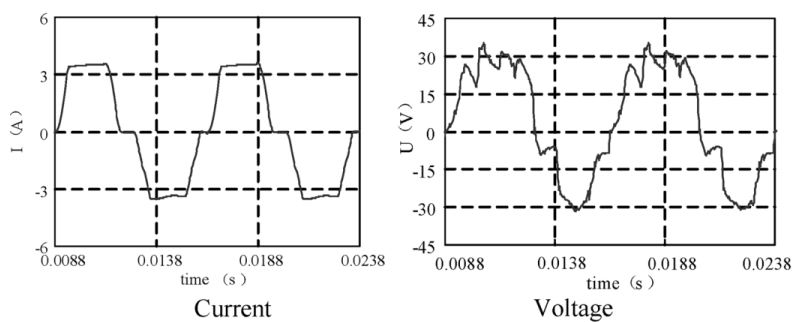

(a)

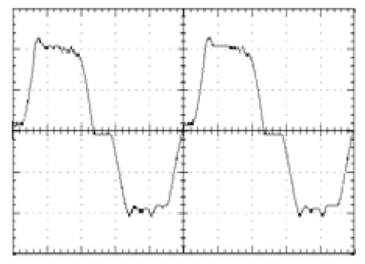

Current (1.5A/div)



Voltage (15V/div)

(b)

Fig. 7. Phase current and voltage at $1000 \mathrm{r} / \mathrm{min}$. (a) Simulated. (b) Measured.

TABLE I

The dC Output Voltage $U_{\mathrm{dc}}$ AND dC Field Current $I_{f}$ Versus Speed

\begin{tabular}{c|c|ccccc}
\hline \hline \multicolumn{2}{c|}{ S peed (rpm) } & 400 & 800 & 1000 & 1200 & 1600 \\
\hline \multirow{2}{*}{$U_{D C}(\mathrm{~V})$} & Simulated & 63.9 & 62.6 & 63.3 & 64.0 & 64.1 \\
& Measured & 63.1 & 63.2 & 63.9 & 62.6 & 63.4 \\
\hline \multirow{2}{*}{$I_{f}(\mathrm{~A})$} & Simulated & +3.83 & +1.36 & 0 & -1.21 & -3.70 \\
& Measured & +3.76 & +1.47 & 0 & -1.18 & -3.54 \\
\hline \hline
\end{tabular}

field excitations. Fig. 6 illustrates the flux control capability versus dc field MMF.

Because the back-EMF of phase winding is proportional to the flux density (or flux), hence, (1) is equivalent to

$$
\alpha=\left(e_{\mathrm{rms}}-e_{\mathrm{rms} 0}\right) / e_{\mathrm{rms} 0} \times 100 \%
$$

where $e_{\mathrm{rms}}$ is the root-mean-square (rms) value of the output voltage, and $e_{\mathrm{rms} 0}$ is the one with no dc field current. To validate experimentally the cosimulated flux control capability, the back-EMFs at different dc field currents at $1000 \mathrm{r} / \mathrm{min}$ are measured and the flux control capability is obtained by using (2), which is plotted in Fig. 6. Moreover, for comparison, the flux control capability from the equivalent magnetic circuit analysis is also shown in Fig. 6 [4]. It can bee seen that the cosimulated result is much closer with the measurement than the magnetic circuit analysis results due to the fact that the eddy current effects, saturation, motion effects, and losses are considered in the cosimulation.

By using the integrated finite element/circuit model shown in Fig. 3, the HEDS machine is cosimulated as a generator over a wide speed range. The voltage of the capacitance can be controlled as a constant at the rated conditions by a proportional and integral (PI) regulation. By online-tuning the dc field current, the dc output voltage can be maintained constant over a wide range of rotor speeds. Fig. 7 shows the waveforms of the simulated and measured phase current and voltage at $1000 \mathrm{r} / \mathrm{min}$. It can be found that the waveforms using cosimulated method closely match the measured ones. Table I lists the dc output voltage and $\mathrm{dc}$ field currents at different speeds. Both cosimulation and measurement show that the proposed machine can output a constant voltage over a wide speed range with the use of flux control.



Fig. 8. Efficiency comparison between HEDS and SDFDS machines.

Based on the cosimulation method, the efficiency of both the HEDS machine and SDFDS machines over the whole speed region is obtained as shown in Fig. 8. In the low-speed and rated region, the HEDS machine inevitably needs less field winding MMF to realize the desired flux linkage, resulting in higher efficiency than the SDFDS machine. In high-speed region, however, the HEDS machine needs relatively higher field winding MMF to realize flux weakening, resulting in lower efficiency than the SDFDS machine.

\section{CONClusion}

In this paper, a HEDS machine for automotive engines has been analyzed. Because of its distinct capability of flux control, the proposed machine can realize a very wide range (four times) of flux regulation, hence, achieving a high starting torque for cold cranking and maintaining a constant output voltage for battery charging over a wide speed range. These two features are highly desirable for the machine to work as an ISG for modern automobiles and HEVs.

The newly proposed cosimulation approach allows FEA and the circuit simulator to work simultaneously, and provides a tight integration and seamless data exchange capability in system level. Experimental results on the prototype machine have been given to verify the technique. The method is not only more efficient and flexible, but also more accurate and stable. It can readily be extended to other machine and drive systems.

\section{ACKNOWLEDGMENT}

This work was supported by the National Natural Science Foundation of China (NSFC) under Grants 50337030 and 50377004.

\section{REFERENCES}

[1] C. Williams, "Comparison and review of electric machines for integrated starter generator applications," in Proc. IEEE IAS Аnпи. Meeting, 2004, vol. 1, pp. 386-396.

[2] K. T. Chau, M. Cheng, and C. C. Chan, "Nonlinear magnetic circuit analysis for a novel stator-doubly-fed doubly-salient machine," IEEE Trans. Magn., vol. 38, no. 5, pp. 2382-2384, Sep. 2002.

[3] A. Monnier, B. Froidurot, C. Jarrige, R. Meyer, and P. Teste, "A mechanical, electrical, thermal coupled-field simulation of a sphere-plane electrical contact," in Proc. IEEE Holm Conf. Electr. Contacts, 2005, pp. 224-231.

[4] X. Zhu and M. Cheng, "Design and analysis of a new hybrid excited doubly salient machine capable of field control," in Proc. IEEE IAS Аnnu. Meeting, 2006, vol. 5, pp. 2382-2389.

Manuscript received October 30, 2006; revised February 5, 2007 (e-mail: mcheng@seu.edu.cn). 